\title{
A qPCR and multiplex pyrosequencing assay combined with automated data processing for rapid and unambiguous detection of ESBL-producers Enterobacteriaceae
}

\author{
Yann Deccache ${ }^{1}$, Leonid M Irenge ${ }^{1}$, Jérôme Ambroise², Encho Savov ${ }^{3}$, Dan Marinescu ${ }^{4}$, \\ Raphael B Chirimwami ${ }^{5}$ and Jean-Luc Gala ${ }^{1,2^{*}}$
}

\begin{abstract}
Rapid and specific detection of extended-spectrum $\beta$-lactamase-producing (ESBL) bacteria is crucial both for timely antibiotic therapy when treating infected patients as well as for appropriate infection control measures aimed at curbing the spread of ESBL-producing isolates. Whereas a variety of phenotypic methods are currently available for ESBL detection, they remain time consuming and sometimes difficult to interpret while being also affected by a lack of sensitivity and specificity. Considering the longer turnaround time (TAT) of susceptibility testing and culture results, DNA-based ESBL identification would be a valuable surrogate for phenotypic-based methods. Putative ESBL-positive Enterobacteriaceae isolates $(n=330)$ from clinical specimen were prospectively collected in Bulgaria, Romania and Democratic Republic of Congo and tested in this study. All isolates were assessed for ESBL-production by the E-test method and those giving undetermined ESBL status were re-tested using the combination disk test. A genotypic assay successively combining qPCR detection of blaCTX-M, blaTEM and blaSHV genes with a multiplex pyrosequencing of blaTEM and blaSHV genes was developed in order to detect the most common ESBL-associated TEM and SHV single nucleotides polymorphisms, irrespective of their plasmid and/or chromosomal location. This assay was applied on all Enterobacteriaceae isolates $(n=330)$. Phenotypic and genotypic results matched in 324/330 (98.2\%). Accordingly, real-time PCR combined with multiplex pyrosequencing appears to be a reliable and easy-to-perform assay with high-throughput identification and fast TAT ( $\sim 5 h)$.
\end{abstract}

Keywords: ESBLs, Detection, $\mathrm{qPCR}$, Multiplex pyrosequencing

\section{Introduction}

Beta-lactams are antimicrobial drugs sharing a $\beta$-lactam ring structure (Kong et al. 2009). They are among the most commonly prescribed drugs worldwide, especially for treatment of infections caused by Gram-negative bacteria (GNB) (Poole 2004; Paterson 2006; Pitout et al. 1997). A major shortcoming of this massive use of $\beta$-lactams antibiotics is the sharp and steady increase of

\footnotetext{
*Correspondence: jean-luc.gala@uclouvain.be

${ }^{2}$ Center for Applied Molecular Technologies, Institut de Recherche Expérimentale et Clinique, Université Catholique de Louvain, B1.30.24, Clos Chapelle-aux-Champs, 1200 Brussels, Belgium

Full list of author information is available at the end of the article
}

antimicrobial resistance as evidenced worldwide over the past decades (Pitout and Laupland 2008; Gibold et al. 2014). The main resistance mechanism is the synthesis of $\beta$-lactamases which retain the ability of hydrolyzing the $\beta$-lactam ring of a wide range of $\beta$-lactam antibiotics, including penicillins, cephalosporins and carbapenems (Drawz and Bonomo 2010; Bush 2010). These hydrolyzing enzymes are encoded by genes located either on bacterial chromosomes or on extra-chromosomal transferable mobile elements (Poole 2004). So far, more than 1,300 $\beta$-lactamases have been described (Bush 2013) (http://www.lahey.org/studies/) and divided in four molecular classes (A-D) depending on their amino acids sequence (Jacoby and Munoz-Price 2005).

\section{Springer}


Class A $\beta$-lactamases, which is mainly found in GNB, is the most prevalent worldwide. Among GNB, extended spectrum $\beta$-lactamases (ESBLs) are the most common as illustrated by ESBL-producing Enterobacteriaceae which have emerged as an important cause of a wide spectrum of infections (Giamarellou 2005; Owens and Lautenbach 2008). ESBLs confer resistance to penicillins, cephalosporins (first-, second- and third-generation), and aztreonam while retaining susceptibility to clavulanate (Paterson and Bonomo 2005). Many ESBLs are derived from $\beta$-lactamase variants arising from amino acids substitutions that enable enzymes to hydrolyze various $\beta$-lactam antibiotics (Bradford 2001; Haanperä et al. 2008). Among class A ESBL families, the two main representatives are TEM and SHV in which amino acids substitutions occur in blaTEM and blaSHV genes (Pfaller and Segreti 2006). In fact, some isolates may carry concomitantly a chromosomal $\beta$-lactamase (most commonly SHV-1 or TEM-1) and the plasmid variants derived from the chromosomal blaSHV or blaTEM gene. This chromosomal $\beta$-lactamase may interfere with molecular detection of the ESBL gene usually residing in plasmids (Haanperä et al. 2008; Jones et al. 2009).

Another major ESBL family is CTX-M which derives from Klyuvera spp (Rossolini et al. 2008). The successful spread of ESBLs in a wide range of Enterobacteriaceae can be attributed to the fact that the genes coding for ESBLs are often located on self-transmissible or mobilizable broad-host-range plasmids (Livermore et al. 2007; D'Andrea et al. 2013).

Extended-spectrum $\beta$-lactamase detection is crucial both for infection control measures as well as for selecting appropriate antimicrobial therapy, as failure to rapidly and unambiguously identify ESBL-producing isolates may delay the initiation of appropriate infection control measures and further contribute to their spread in hospital and community settings (Bradford 2001).

In the majority of healthcare facilities, the routine detection of ESBL production by Enterobacteriaceae is actually carried out by phenotypic methods (Pitout and Laupland 2008). These tests are based on the principle that most ESBLs hydrolyze third-generation cephalosporins and are inhibited by clavulanic acid (Robin et al. 2008; Jarlier et al. 1988; Cormican et al. 1996; Carter et al. 2000). Most guidelines recommend to first screen isolates for decreased susceptibility to extended-spectrum cephalosporins in primary susceptibility testing, and then to carry out a confirmatory test to verify ESBL production (Gazin et al. 2012; Garrec et al. 2011; Rupp and Fey 2003). However, and despite the variety of phenotypic methods available and the existence of various guidelines available for the phenotypic detection of ESBL-producing bacteria, this remains a contentious issue, and proficiency testing shows that compliance varies widely across different parts of the world (Pitout and Laupland 2008). For instance, it has been reported that laboratories fail to interpret correctly the inhibition ellipse in $30 \%$ of cases. Indeed, the enzymes may vary in their substrate affinities and in their catalytic efficiencies, and also differ in their penetration rates into bacterial cells (Drieux et al. 2008). Furthermore, the emergence of AmpC $\beta$-lactamases complicates the detection of ESBL-production. Indeed, AmpC $\beta$-lactames display high resistance rate to cephalosporins without retaining any susceptibility to clavulanate (Polsfuss et al. 2011). This observation is important, considering the recent emergence of plasmidic AmpC $\beta$-lactamases species like Escherichia coli and Klebsiella spp (Jacoby 2009).

In that respect, DNA-based detection of ESBL-production appears as a valuable alternative to the phenotypic-based methods. It is indeed independent of gene expression and relatively rapid as compared with susceptibility testing and culture results. In addition, this type of assay could help unravel issues related to ESBL-producing isolates, among which false-negative or indeterminate results.

Several molecular tests aiming to detect and/or characterize TEM, SHV and CTX-M have previously been described (Naas et al. 2007; Haanperä et al. 2008; Oxacelay et al. 2009; Jones et al. 2009; Leinberger et al. 2010; Cohen Stuart et al. 2010; Al-Agamy et al. 2014). However, despite these encouraging achievements, technological limitations still hamper their implementation in routine microbiology practice, mainly due to the fact that they are still cumbersome and time-consuming (Drieux et al. 2008; Wintermans et al. 2013). Consequently, there is still a need for molecular high-throughput techniques detecting rapidly and reliably ESBL-producing isolates.

The aim of the current study was to develop and test a multiplex pyrosequencing assay for quick and unambiguous detection of ESBL-producing Enterobacteriaceae isolates. In this work, real-time PCR amplification of blaCTX-M, blaSHV and blaTEM genes was completed by multiplex pyrosequencing characterization of blaTEM and blaSHV in order to detect ESBL associated variants in isolates from Bulgaria, Romania and Democratic Republic of Congo.

\section{Materials and methods \\ Bacterial isolates}

Various clinical specimens [including blood culture, peritoneal, pleural and pericardial fluids, urine, pus and sputum; n = 330)] were collected between 2008 and 2014 from patients from Bucharest Clinical Emergency Hospital (Romania; $n=177$ ), from the Intensive Care Unit (ICU) at the Military Medical Academy of Sofia 
(Bulgaria; $\mathrm{n}=144$ ) and from various hospital wards of the Bukavu Provincial General Hospital (South Kivu province, Democratic Republic of Congo; $\mathrm{n}=9$ ). After initial culture, presumptive Enterobacteriaceae isolates from DR Congo were identified using standard microbiological methods whereas isolates from Bulgaria and Romania were identified using the Vitek-2 automated instrument ID system (BioMérieux, Marcy l'Etoile, France). Four commercial strains were used as negative and positive controls: ATCC-35218 (non-ESBL producing E. coli), ATCC-700603 (SHV-18 type $\beta$-lactamase producing $K$. pneumoniae), DSM-22314 (TEM-46 ESBL producing E. coli), and DSM-22313 (TEM-50 ESBL producing E. coli), (see Table 1).

\section{Susceptibility testing}

In Romania and Bulgaria, ESBL-production assay was performed using Vitek-2 cards whereas in DRC, it was carried out by the double-disk synergy test on Mueller-Hinton II ${ }^{\circledR}$ Agar (Oxoid Ltd, Cambridge, UK) using disks soaked with ceftazidime or cefotaxime ( $30 \mu \mathrm{g}$ each) placed at a distance of $20 \mathrm{~mm}$ apart from a disk containing amoxicillin plus clavulanic acid $(10 \mu \mathrm{g})$. A clearcut enhancement of the inhibition in front of either ceftazidime and cefotaxime disks towards the clavulanic acid-containing disk (also called "champagne-cork" or "keyhole") was interpreted as positive for ESBL production (Drieux et al. 2008).

E-test ${ }^{\circledR}$ strips (BioMérieux, Marcy l'Etoile, France) were used for confirmation of ESBL-production. Minimum inhibitory concentrations (MIC) of ceftazidime and cefotaxime with and without clavulanic acid were determined after 16-18 $\mathrm{h}$ incubation on ISO Sensitest ${ }^{\circledR}$ Agar plates inoculated with suspension of isolates at a fixed density (0.5-0.6 McFarland standard). The test was performed and interpreted according to the manufacturer's instructions. Escherichia coli ATCC 35218 and Klebsiella pneumoniae ATCC 700603 strains were used as ESBL negative and positive controls, respectively.

Each isolate generating a non-determinate (ND) result with this gradient test was retested using the combination disk test (CDT) as described by Carter (Carter et al. 2000). In brief, disks containing $30 \mu \mathrm{g}$ of cefotaxime, ceftazidime or cefepime and disks containing a combination of each of these drugs with $10 \mu \mathrm{g}$ clavulanic acid (BioRad, Nazareth Eke, Belgium) were placed independently on a 0.5 McFarland opacity lawn culture of the tested isolate on a ISO Sensitest ${ }^{\circledR}$ Agar plate and incubated for $18-24 \mathrm{~h}$ at $37^{\circ} \mathrm{C}$. Isolates were considered ESBL-producing if the inhibition zone measured around one of the combination disks was at least $5 \mathrm{~mm}$ larger that of the corresponding cephalosporin disk.

\section{DNA extraction and DNA-based identification of isolates}

For each isolate, a single colony was selected after overnight growth on ISO-Sensitest ${ }^{\circledR}$ agar plate and cultured in fresh ISO-Sensitest ${ }^{\circledR}$ broth (Oxoid Ltd, Cambridge, UK) for $6 \mathrm{~h}$. The broth was centrifuged and DNA subsequently extracted from the pelleted cells using a DNA tissue kit with on the BioRobot EZ1 (Qiagen, Hilden, Germany) according to the manufacturer's instructions.

Purified DNA was quantified using NanoDrop ND1000 ${ }^{\circledR}$ spectrophotometer (Nanodrop Technologies, Inc. Wilmington, DE, US) and stored at $-20^{\circ} \mathrm{C}$ for further molecular assays. In case of ambiguous species identification with the Vitek-2 system, DNA-based identification was then performed as described previously (Bosshard et al. 2006; Vandercam et al. 2008).

\section{Polymerase chain reaction (PCR) amplification and pyrosequencing assay \\ Primers design}

All publicly available nucleotide sequences of blaTEM and blaSHV alleles related to ESBL-production phenotype (http://www.lahey.org/Studies/) were retrieved from the GenBank nucleotide sequence database and aligned using the BioNumerics software v7.1. (Applied Maths, Sint-Martens-Latem, Belgium). A total of 88 blaTEM and 30 blaSHV nucleotide sequences were aligned. Consequently, two simplex qPCRs were designed to amplify a 302 bp sequence flanking amino acids 179, 238 and 240 of blaSHV and a 500 bp sequence flanking positions 104, 164 and 238 of blaTEM (Table 2). The aforementioned positions include indeed the most frequent substitutions associated with ESBL-production phenotype (Cohen Stuart et al. 2010). A duplex qPCR was used to amplify two blaCTX-M fragments of 224 and 175 bp as previously described (Naas et al. 2007).

Table 1 Commercial reference strains used as negative and positive controls

\begin{tabular}{|c|c|c|c|c|c|c|c|}
\hline & TEM $_{104}$ & TEM $_{164}$ & TEM $_{238}$ & $\mathrm{SHV}_{179}$ & $\mathrm{SHV}_{238}$ & $\mathrm{SHV}_{240}$ & Phenotype \\
\hline ATCC-35218 (E. coli) & $\mathrm{Glu}_{104}$ & $\operatorname{Arg}_{164}$ & Gly $_{238}$ & - & - & - & Non-ESBL \\
\hline ATCC-700603 (K. pneumoniae) & - & - & - & Asp $_{179}$ & Gly $_{238}$ Ala & Glu $_{240}$ Lys & SHV-18 \\
\hline DSM-22313 (E. coli) & Glu $_{104}$ Lys & $\operatorname{Arg}_{164}$ & $\mathrm{Gly}_{238} \mathrm{Ser}$ & - & - & - & TEM-46 \\
\hline DSM-22314 (E. coli) & Glu $_{104}$ Lys & $\operatorname{Arg}_{164} \mathrm{Ser}$ & Gly $_{238}$ & - & - & - & TEM-50 \\
\hline
\end{tabular}


Table 2 List of primers used for ESBL-encoding gene detection and pyrosequencing in Enterobacteriaceae

\begin{tabular}{|c|c|c|c|c|c|}
\hline Target gene & Primer name & Sequence $5^{\prime}-3^{\prime}$ & qPCR & Pyrosequencing assay & Reference \\
\hline \multirow[t]{5}{*}{ blaTEM } & TEM-Forward & biotin-CGCCGCATACACTATTCTCA & \multirow[t]{5}{*}{ Simplex } & \multirow[t]{5}{*}{ Triplex } & \multirow[t]{5}{*}{ Own primer design } \\
\hline & TEM-Reverse & ATACGGGAGGGCTTACCATC & & & \\
\hline & TEM-seq104 & TGCTTTTCTGTGACTGGTG & & & \\
\hline & TEM-seq164 & TTCAGCTCCGGTTCC & & & \\
\hline & TEM-seq238 & CGCKAGAHCCACGCT & & & \\
\hline \multirow[t]{4}{*}{ blaSHV } & SHV-Forward & biotin-TCTGYGCCGCCGTCATTA & \multirow[t]{4}{*}{ Simplex } & \multirow[t]{4}{*}{ Duplex } & \multirow[t]{4}{*}{ Own primer design } \\
\hline & SHV-Reverse & CTTTGTTATTCGGGCCAAGCA & & & \\
\hline & SVH-seq179 & GCTGGCCGGGGATGTG & & & \\
\hline & SVH-seq238 & ATSCCGCGCSCACCCC & & & \\
\hline \multirow[t]{4}{*}{ blaCTX-M } & CTX-1Forward & ATGTGCAGYACCAGTAARGTGA & \multirow[t]{4}{*}{ Duplex } & \multirow[t]{4}{*}{-} & \multirow[t]{4}{*}{ Naas et al. (2007) } \\
\hline & CTX-1Reverse & TGRGMAATCARYTTRTTCAT & & & \\
\hline & CTX-2Forward & TGGTRAYRTGGMTBAARGG & & & \\
\hline & CTX-2Reverse & TGGGTRAARTARGTSACCAGAA & & & \\
\hline
\end{tabular}

Degenerate base symbols (IUPAC code): $K$ represents $G / T, Y$ is $T / C, S$ is $G / C, R$ is $G / A, M$ is $A / C, H$ is $A / T / C$ and $B$ is $G / T / C$.

\section{Simplex and duplex qPCR amplification}

Each qPCR was carried out in $50 \mu \mathrm{L}$ of a reaction mixture containing $100 \mathrm{pg}$ of extracted DNA as template, $300 \mathrm{nM}$ of each primer, and $25 \mu \mathrm{L}$ of Power SYBR ${ }^{\circledR}$ Green Reagents 2x (Applied Biosystems, Nieuwekerk, The Netherlands). Amplifications were performed either on a 7900HT Fast Real-Time PCR System (Applied Biosystems, Nieuwekerk, The Netherlands) or a Stratagene Mx3005P (Agilent Technologies, Santa Clara, CA, USA). The reaction was initiated at $50^{\circ} \mathrm{C}$ for $2 \mathrm{~min}$ and $95^{\circ} \mathrm{C}$ for $10 \mathrm{~min}$ followed by 45 cycles at $95^{\circ} \mathrm{C}$ for $15 \mathrm{~s}$ and $63^{\circ} \mathrm{C}$ for 1 min for the TEM and SHV qPCRs. The duplex CTX-M was carried out using the same conditions as above except that the optimal annealing temperature was $57^{\circ} \mathrm{C}$. Finally, a melting curve analysis was performed. Data were analyzed using the analytical software SDS 2.4 from Applied BioSystem or MxPro qPCR software from Agilent Technologies.

\section{Multiplex pyrosequencing protocol}

Pyrosequencing is a bioluminometric assay allowing rapid and high throughput sequencing of short DNA sequences. This type of assay has already proved it value for assessing drug-resistance determining regions in various bacteria (Deccache et al. 2011; Haanperä et al. 2005). In order to increase the throughput of the assay, two multiplex pyrosequencing assays were concomitantly developed using three and two pyrosequencing primers (Table 2) to generate corresponding sequences for blaTEM and blaSHV amplicons, respectively.

The pyrosequencing assay was performed using a PyroGold SQA sample preparation kit (Qiagen, Hilden, Germany) according to manufacturer's instructions. Briefly, $40 \mu \mathrm{l}$ of biotinylated PCR products were immobilized for
15 min using $4 \mu \mathrm{l}$ streptavidin-coated Sepharose beads (GE Healthcare-Biosciences AB, Uppsala, Sweden) and captured using the Vacuum Prep Tool (Qiagen, Hilden, Germany) according to manufacturer's instructions. After a denaturation step $(\mathrm{NaOH} 0.5 \mathrm{M}$ during $5 \mathrm{~s})$ and a wash step for the removal of unlabeled complementary DNA of the amplicon, the biotinylated single-stranded DNA present on the streptavidin-coated Sepharose beads were transferred to a 96-well plate and used as template for the pyrosequencing assay with a mixture of sequencing primers. The TEM mix contained 6, 20 and 60 pmol of the TEM-seq104, TEM-seq164 and TEM-seq238 primers, respectively, whilst the SHV mix contained 60 and 20 pmol of the SHV-seq179 and SHV-seq238 primers, respectively. The pyrosequencing nucleotide dispensation orders were selected using SENATOR (SElecting the Nucleotide dispensATion Order) algorithm (Ambroise et al. 2013). Nucleotide dispensation orders with 15 (CAGCCTGACATATCA) and 11 (GTGACTGCGTC) nucleotides were selected for the triplex TEM and duplex SHV assays, respectively. Pyrosequencing was carried out using a PSQ 96 MA system (Qiagen, Hilden, Germany).

All multiplex pyrosequencing signals generated for TEM and SHV were analyzed using a new web interactive application (available at https://ucl-irec-ctma.shinyapps.io/Pyrosequencing-TEM-SHV and in 'download' section of http://www.uclouvain.be/ctma.html where a template data set can be downloaded) written with R.3.1.2 software (Ihaka and Gentleman 1996) using the "shiny" package. This new application was developed using an improved version of three previously published algorithms including (1) AdvISER-PYRO for analyzing low and complex signals resulting from samples including several mycobacteria (Ambroise et al. 2013), 
(2) AdvISER-M-PYRO for analyzing overlapping pyrosignals generated from multiplex reactions conducted on mono-allelic genes in bacteria (Ambroise et al. 2014), and (3) AdvISER-MH-PYRO for analyzing overlapping pyro-signals generated from multiplex reactions to genotype bi-allelic human SNP (Ambroise et al. 2015). While the initial version of AdvISER-M-PYRO analyzed multiplex pyrosequencing signals by selecting a single and unique sequence for each genomic region, this new version enables the analysis of multiplex pyrosequencing signals generated from samples including two distinct sequences (i.e., plasmid and chromosomal) for each genomic region.

The new web interactive application enables also the integration of PCR and pyrosequencing results in order to correctly identify the ESBL status of each isolate. In that respect, an isolate was considered as ESBL-producer organism if either one or more of the ESBL-associated substitutions was detected from pyrosequencing signals of blaTEM or blaSHV, or if the blaCTX-M gene was present (as illustrated on Fig. 1). On the contrary, the isolate was considered as non-ESBL-producer organism when blaTEM, blaSHV and blaCTX-M genes were either not detected or when amplified blaSHV or blaTEM genes did not carry any ESBL-associated substitution. Pyrosequencing signals displaying low signal-to-noise ratio (maximum peak height $<10$ relative fluorescence units) and/or a low confidence index $(\mathrm{R}<0.99)$ were considered as unsafe and re-tested using the same protocol.

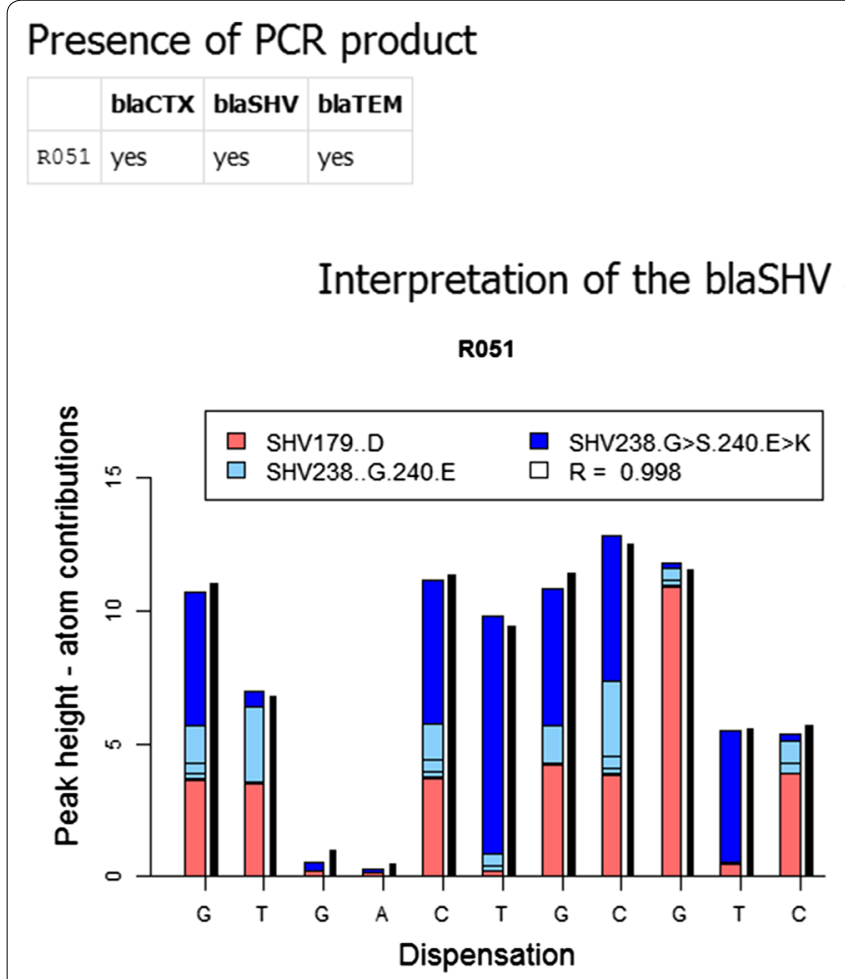

R051

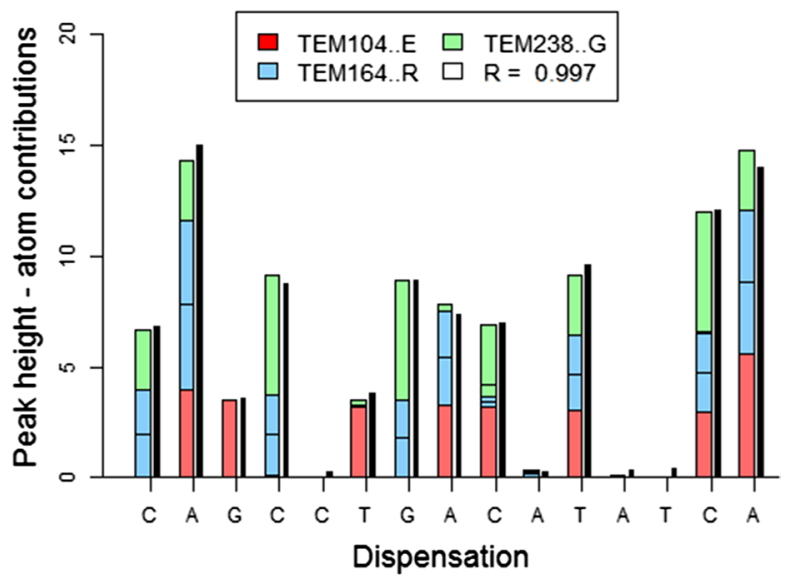

Conclusion

\section{Isolate R051 is considered as ESBL-producer because : \\ - a blaCTX-M gene is detected \\ - an ESBL-associated substitution is detected in blaSHV}

Fig. 1 Output illustration of the web interactive application. For a given isolate, integration of PCR and multiplex pyrosequencing results allows the determination of the ESBL-producing status (available at https://ucl-irec-ctma.shinyapps.io/Pyrosequencing-TEM-SHV/). As demonstrated in this example, the algorithm enables to correctly decompose the multiplex pyrosequencing signal generated for the blaSHV gene despite the presence of two sequences for the blaSHV238-240 genomic region (i.e., G238 and E240 on one variant and the G238S-E240K double substitutions on another one). 


\section{Sequencing analysis of amplicons}

Sanger sequencing was used to confirm pyrosequencing results. Amplifications products of blaTEM and blaSHV were purified using $\mathrm{MSB}^{\circledR}$ Spin PCRapace purification kit (STRATEC Molecular GmbH, Berlin, Germany) according to manufacturer's instructions. After having assessed $5 \mu \mathrm{l}$ of each purified PCR product on a $2 \%(\mathrm{w} / \mathrm{v})$ agarose gel stained with ethidium bromide, corresponding amplicons were sequenced in both directions on an automated ABI 3130 Genetic Analyser apparatus (Applied Biosystems, Nieuwekerk, The Netherlands), using the BigDye Terminator v3.1 Cycle Sequencing kit from the same manufacturer. Nucleotide and deduced amino acid sequences were analyzed using the BioNumerics software v7.1. (Applied Maths, Sint-Martens-Latem, Belgium) and compared to publically available database.

\section{Results}

Phenotypic identification of all isolates $(\mathrm{n}=330)$ was as follows: Klebsiella pneumonia $(\mathrm{n}=147)$, Escherichia coli $(\mathrm{n}=119)$, Klebsiella spp. $(\mathrm{n}=30)$, Enterobacter cloacae $(\mathrm{n}=16)$, Proteus mirabilis $(\mathrm{n}=10)$, Serratia marcescens $(\mathrm{n}=6)$, and Morganella morgannii $(\mathrm{n}=2)$.

\section{Phenotypic results}

ESBL- and non-ESBL-producing organisms were assessed by E-test assay in 267/330 (80.9\%) and 20/330 (6.1\%) isolates, respectively whereas $43 / 330$ (13\%) presented a MIC value lower than the predefined range, hence yielding an ND result. CDT was used to retest these 43 isolates displaying a ND E-test, whereupon 36 isolates were found ESBL-producers and 7 non-ESBL producers.

\section{Genotypic results}

The newly-developed multiplex pyrosequencing assay was used to rapidly assess the ESBL-producing status of all isolates following blaTEM, blaSHV and blaCTX-M amplification by qPCR. Among all isolates $(\mathrm{n}=330), 295$ $(89.4 \%)$ produced high-quality pyrosequencing signals (maximum peak height $>10, \mathrm{R}>0.99$ ) while $35(10.6 \%)$ isolates did not meet these quality criteria and where therefore re-tested with the same protocol. After this second round of analysis, 308 (93.3\%) and 22 (6.7\%) isolates were identified as ESBL- and non-ESBL-producing bacteria, respectively.

The TAT for the analysis was $\sim 5 \mathrm{~h}$ (i.e., $40 \mathrm{~min}$ for DNA purification, $1 \mathrm{~h} 50$ for the duplex qPCR blaCTX-M, $1 \mathrm{~h} 50$ for the concomitant simplex TEM and SHV qPCR, and $40 \mathrm{~min}$ for the multiplex pyrosequencing and data processing using the new web interactive application).

It is of note that the Sanger sequence analysis of all blaSHV and blaTEM amplification fragments was perfectly concordant with the pyrosequencing results (data not shown).

\section{Phenotypic: genotypic comparison}

Comparison between DNA-based and E-test results is detailed in Table 3. When CDT test was combined with E-test, a quasi-perfect concordance with results from the multiplex DNA-assay was observed (Table 4). Altogether after CDT retesting, six results remained discordant: one P. mirabilis and four E. coli isolates considered as 'nonESBL producing bacteria' by CDT despite carrying the blaCTX-M gene (Table 4). Conversely, R080 isolate was considered as 'ESBL-producing bacteria' according to E-test, while neither blaSHV, blaTEM, nor blaCTX-M genes were detected by qPCR. The ESBL phenotype was however confirmed after re-testing this isolate with CDT.

It is of note that another discordant result was observed between E-test and DNA-based test on E. cloacae MMA55 isolate. This isolate was characterized as 'nonESBL producing bacteria according to the E-test, despite the presence of a blaSHV gene with point mutations leading to a double $\mathrm{E}_{240} \mathrm{~K}$ and $\mathrm{G}_{238} \mathrm{~S}$ ESBL-associated substitution. CDT confirmed however the status of ESBLproduced. The isolate should therefore not be considered as a phenotypic-genotypic discordant.

\section{Discussion}

A new DNA-based assay has been developed for enabling rapid and unambiguous detection of ESBL-producing Enterobacteriaceae isolates. The method consists of a qPCR amplification of blaTEM, blaSHV and blaCTX$\mathrm{M}$ and subsequent multiplex pyrosequencing of ESBL production-related mutations within blaSHV and blaTEM genes. All selected mutations have previously been reported (Naas et al. 2007; Haanperä et al. 2008; Oxacelay et al. 2009; Jones et al. 2009; Leinberger et al. 2010; Cohen Stuart et al. 2010; Al-Agamy et al. 2014). Rapid and unambiguous detection methods for ESBLproduction are paramount for appropriate management of infectious diseases as it has long been recognized that prompt and targeted antibiotic therapy improves the outcome for patients with infectious diseases (Perez et al. 2007). Furthermore, rapid laboratory detection is critical for the development of infection control measures aimed at reducing the transmission rate of antibiotic-resistant microorganisms in hospital wards (Stürenburg and Mack 2003). Up to now, phenotypic methods have routinely been used for detecting antimicrobial resistant isolates, and particularly ESBL-producing isolates (Robin et al. 2008; Jarlier et al. 1988; Cormican et al. 1996; Carter et al. 2000). Unfortunately, recent studies have consistently pinpointed the many drawbacks affecting these methods. These include a long TAT (24-72 h) and somehow low 
Table 3 Distribution of the 330 isolates tested, depending on the species, phenotypic test (E-test) and DNA-based test

\begin{tabular}{|c|c|c|c|c|c|c|}
\hline & \multirow[t]{2}{*}{ E-test } & \multicolumn{4}{|c|}{ DNA-based test } & \multirow[t]{2}{*}{$\mathrm{n}$} \\
\hline & & CTX-M & TEM & SHV & Interpretation & \\
\hline \multirow[t]{6}{*}{ E. cloacae, $n=16$} & ESBL & Absent & Wild-type & Mutated & ESBL & 1 \\
\hline & & Present & Absent & Absent & ESBL & 1 \\
\hline & & & Wild-type & Absent & ESBL & 8 \\
\hline & ND & Absent & Wild-type & Mutated & ESBL & 1 \\
\hline & & Present & Wild-type & Absent & ESBL & 4 \\
\hline & Non-ESBL & Absent & Wild-type & Mutated & ESBL* & $1^{*}$ \\
\hline \multirow[t]{6}{*}{ E. coli, $n=119$} & ESBL & Present & Absent & Absent & ESBL & 34 \\
\hline & & & Wild-type & Absent & ESBL & 50 \\
\hline & & & & Mutated & ESBL & 2 \\
\hline & ND & Present & Wild-type & Absent & ESBL & 20 \\
\hline & Non-ESBL & Absent & Absent & Absent & Non-ESBL & 3 \\
\hline & & Absent & Wild-type & Absent & Non-ESBL & 10 \\
\hline \multirow[t]{14}{*}{ K. pneumoniae, $n=147$} & ESBL & Absent & Absent & Absent & Non-ESBL* & $1^{*}$ \\
\hline & & & Wild-type & Mutated & ESBL & 8 \\
\hline & & Present & Absent & Wild-type & ESBL & 4 \\
\hline & & & Wild-type & Absent & ESBL & 4 \\
\hline & & & & Wild-type & ESBL & 73 \\
\hline & & & & Mutated & ESBL & 41 \\
\hline & ND & Absent & Absent & Absent & Non-ESBL & 2 \\
\hline & & & Wild-type & Mutated & $\mathrm{ESBL}$ & 3 \\
\hline & & Present & Wild-type & Absent & ESBL & 1 \\
\hline & & & & Wild-type & ESBL & 5 \\
\hline & & & & Mutated & ESBL & 1 \\
\hline & Non-ESBL & Absent & Absent & Absent & Non-ESBL & 2 \\
\hline & & & & Wild-type & Non-ESBL & 1 \\
\hline & & & Wild-type & Wild-type & Non-ESBL & 1 \\
\hline \multirow[t]{5}{*}{ Klebsiella spp., $\mathrm{n}=30$} & ESBL & Present & Absent & Wild-type & ESBL & 1 \\
\hline & & & Wild-type & Absent & ESBL & 13 \\
\hline & & & & Wild-type & ESBL & 5 \\
\hline & & & & Mutated & ESBL & 10 \\
\hline & ND & Present & Wild-type & Absent & ESBL & 1 \\
\hline \multirow[t]{6}{*}{ P. mirabilis, $\mathrm{n}=10$} & ESBL & Present & Wild-type & Absent & ESBL & 1 \\
\hline & & & & Wild-type & ESBL & 1 \\
\hline & & & & Mutated & ESBL & 2 \\
\hline & ND & Absent & Wild-type & Mutated & ESBL & 3 \\
\hline & & Present & Absent & Absent & ESBL & 1 \\
\hline & Non-ESBL & Absent & Absent & Absent & Non-ESBL & 2 \\
\hline \multirow[t]{2}{*}{ S. marcescens, $n=6$} & ESBL & Present & Wild-type & Absent & ESBL & 5 \\
\hline & ND & Present & Wild-type & Absent & ESBL & 1 \\
\hline \multirow[t]{2}{*}{ M. morganii, $\mathrm{n}=2$} & ESBL & Present & Absent & Absent & ESBL & 1 \\
\hline & & & Wild-type & Absent & ESBL & 1 \\
\hline
\end{tabular}

* Isolates for which genotypic and phenotypic results are discordant. Both discordant isolates and isolates for which E-test yielded a 'ND'result ( $n=43)$ were re-tested using the combination disk test (CDT).

sensitivity and specificity. As an illustration, Garrec and co-workers (Garrec et al. 2011) compared various phenotypic methods for detecting ESBL production in Enterobacteriaceae and found the sensitivity and specificity of
ESBL-detection using the Vitek-2 to vary between 92 and $95 \%$ in ESBL-producing E. coli isolates while being substantially lower (i.e., 50-79\%) in ESBL-producing K. pneumonia isolates. Lower sensitivity (71\%) and 
Table 4 Distribution of the 45 isolates re-tested using the CDT ( 43 with a ND and 2 with discrepant results between E-test and DNA-based assay)

\begin{tabular}{|c|c|c|c|c|c|c|c|}
\hline & \multicolumn{3}{|l|}{ Phenotypic test } & \multicolumn{3}{|c|}{ DNA-based test } & \multirow[t]{2}{*}{$\mathrm{n}$} \\
\hline & E-test & $C D$ & CTX-M & TEM & SHV & Interpretation & \\
\hline \multirow[t]{3}{*}{ E. cloacae, $n=6$} & ND & ESBL & Absent & Wild-type & Mutated & ESBL & 1 \\
\hline & & ESBL & Present & Wild-type & Absent & ESBL & 4 \\
\hline & Non-ESBL (MMA55)* & $E S B L^{*}$ & Absent & Wild-type & Mutated & $\mathrm{ESBL}^{*}$ & $1^{*}$ \\
\hline \multirow[t]{2}{*}{ E. coli, $\mathrm{n}=20$} & ND & ESBL & Present & Wild-type & Absent & ESBL & 16 \\
\hline & & Non-ESBL* & Present & Wild-type & Absent & $\mathrm{ESBL}^{*}$ & $4^{*}$ \\
\hline \multirow[t]{6}{*}{ K. pneumoniae, $n=13$} & ND & Non-ESBL & Absent & Absent & Absent & Non-ESBL & 2 \\
\hline & & ESBL & Absent & Wild-type & Mutated & ESBL & 3 \\
\hline & & & Present & Wild-type & Absent & ESBL & 1 \\
\hline & & & & & Wild-type & ESBL & 5 \\
\hline & & & & & Mutated & ESBL & 1 \\
\hline & $\mathrm{ESBL}(\mathrm{R080})^{*}$ & $\mathrm{ESBL}^{*}$ & Absent & Absent & Absent & Non-ESBL* & $1^{*}$ \\
\hline Klebsiella spp., $\mathrm{n}=1$ & ND & ESBL & Present & Wild-type & Absent & ESBL & 1 \\
\hline \multirow[t]{2}{*}{ P. mirabilis, $\mathrm{n}=4$} & ND & ESBL & Absent & Wild-type & Mutated & ESBL & 3 \\
\hline & & Non-ESBL* & Present & Absent & Absent & $\mathrm{ESBL}^{*}$ & $1^{*}$ \\
\hline S. marcescens, $n=1$ & ND & $\mathrm{ESBL}$ & Present & Wild-type & Absent & $\mathrm{ESBL}$ & 1 \\
\hline
\end{tabular}

* Isolates for which genotypic and phenotypic results are discordant. Both discordant isolates and isolates for which E-test yielded a 'ND' result ( $n=43$ ) were re-tested using the combination disk test (CDT).

specificity (73\%) were also reported when using the E-test for detecting ESBL-production in Enterobacteriaceae isolates (Garrec et al. 2011). Moreover, E-test method often yields ND results with some isolates (Leverstein-van Hall et al. 2002). Finally, interpretation of results according to various breakpoints also impacts the interpretation of ESBL-production.

Multiplex DNA-based assays targeting specific mutations related to ESBL-production phenotype are expected to circumvent at least part of these difficulties. Whereas molecular methods are usually considered as exquisitely sensitive and specific for rapidly and directly assessing the ESBL-production status in bacterial isolates, it should be pointed out that few of them have, up to now, convincingly demonstrated their capacity to be usefully translated into the routine practice of clinical microbiology. Consequently, the main goal was to develop a multiplex DNA-based assay which could easily be implemented in a clinical setting, while swiftly delivering a reliable ESBL-status for Enterobacteriaceae isolates. In that respect, qPCR amplification, multiplex pyrosequencing, and signal analysis were combined in order to develop a quick and reliable assay. The signal analysis algorithm was implemented in a new web interactive application that allowed to correctly decomposing triplex and duplex pyrosequencing signals. While a concomitant presence of chromosomal and plasmid $\beta$-lactamase variants within the same isolate does not preclude determining its ESBL phenotype, it may affect the pyrosequencing result of ESBL genes located on the plasmid (Haanperä et al. 2008; Jones et al. 2009). Consequently, the new algorithm was designed to correctly deciphering multiplex pyrosequencing signals generated from samples including two distinct sequences (i.e., plasmid and chromosomal) for each genomic region, as illustrated in Fig. 1.

Compared to phenotypic testing (E-test or CDT), the TAT for the combined multiplex assay was short $(\sim 5 \mathrm{~h})$. In addition, result interpretation is rendered easier and more reproducible by the use of the new web interactive application. To further shorten the TAT, it should also be noted that DNA extraction could be sidestepped while carrying out multiplex amplification directly in triplicate with each isolated bacterial colony, after boiling. Moreover, this multiplex assay appears suitable for simultaneous and high-throughput determination of ESBL-production status on up to 30 isolates per run. Conversely, only 6 isolates could be processed per run if the same test was to be carried out in conventional simplex pyrosequencing format (i.e., one well per pyrosequencing reaction). While the difference between low-cost phenotypic test and DNA-based protocol was important when considering conventional simplex pyrosequencing, the combination of the five uniplex pyrosequencing reactions into two multiplex reactions allowed reducing this gap.

In this study, 43 isolates did not yield MIC values compatible with ESBL-production when using E-test strips. This prompted us to use CDT as complementary test in order to assess and confirm the ESBL-status. As indicated above, 
this process results in extending the TAT which, in turn, could be detrimental for the patient's health. With the latter 43 isolates, the multiplex DNA-based assay gave rapid and unambiguous ESBL characterization. Comparing the results of genotypic and phenotypic tests showed however a discrepant result with five isolates (i.e., four E. coli and one P. mirabilis). In all discrepant cases, E-test and CDT gave an indeterminate result and 'non-ESBL' phenotype, respectively. Considering the presence the blaCTX-M gene in the five isolates, it seems clear that CDT produced false-negative results. According to guidelines published by the European Committee on Antimicrobial Susceptibility Testing (Giske et al. 2013), this type of results may occur when bacteria expressed either a high-level AmpC $\beta$-lactamase (Drieux et al. 2008; Jacoby 2009; Munier et al. 2010), either carbapenemases (such as KPCs or MBLs Tsakris et al. 2009; March et al. 2010) and/or severe permeability defects. The presence of such mechanisms could mask the presence of ESBLs.

The case of MMA55 (E. cloacae) isolate is also interesting as it pinpoints the added value of the current multiplex DNA-based assay compared to phenotypic tests. This isolate was indeed initially characterized as 'nonESBL' by the gradient test even though multiplex qPCR and pyrosequencing revealed the presence of a SHV variant harboring ESBL-associated substitutions $\left(\mathrm{E}_{240} \mathrm{~K}\right.$ and $\mathrm{G}_{238} \mathrm{~S}$ ). Subsequent CDT results were however in line with the ESBL-genotypic result.

Another interesting case was R080 (K. pneumoniae) isolate. Though clearly displaying an ESBL-phenotype, this isolate was lacking the three antibiotic resistance determinant genes as targeted by our qPCR assay. This phenotype could result from the presence of less frequent ESBL genes, such as PER, VEB, SME or BEL. Isolates displaying a similar pattern have indeed previously been described by others (Nijhuis et al. 2012). Investigating these rare genes fell however beyond the immediate scope of this work but investigations will be pursued to understand this apparent discrepancy and to test this hypothesis. By the way, the flexibility of this multiplex pyrosequencing assay makes it perfectly suitable for adding new ESBL family or genetic variant pending their discovery and demonstration of interest.

Like with most DNA-based tests, it must be emphasized that the current assay detects a spectrum of common and highly prevalent ESBL-associated mutations without a precise identification thereof. If this information is required, full-length gene sequencing would need to be carried out for characterization of the genetic variant for point mutations.

One major potential drawback which so far precludes implementing DNA-based methods in routine microbiology is the risk of carry-over contamination. Whereas this risk needs always to be thoroughly assessed, it can be lowered to an acceptable threshold by incorporating UNG-ampErase in PCR mix (Longo et al. 1990) as well as by performing $\mathrm{qPCR}$ preparations and pyrosequencing reactions in separate rooms.

In conclusion, the assay developed in this study can be considered as a bona fide surrogate for ESBL-detection in clinical settings. Moreover, the current multiplex pyrosequencing assay is an innovative high-throughput; rapid and reliable assay drastically limiting the use of DNA while reducing analytical costs and waste material disposal.

\section{Authors' contributions}

YD designed and performed experiments, analyzed data and drafted the manuscript. LMI supervised the work, gave conceptual advices, collected the African strains, and drafted the manuscript. JA developed analytical tool and drafted the manuscript. ES, DM and RBC provided the isolates and critical revision of the manuscript. JLG proposed the subject of the study, organized the network for strain collection in Europe, and thoroughly revised the manuscript. All authors read and approved the final manuscript.

\section{Author details}

${ }^{1}$ Defence Laboratories Department, Belgian Armed Forces, Brussels, Belgium. ${ }^{2}$ Center for Applied Molecular Technologies, Institut de Recherche Expérimentale et Clinique, Université Catholique de Louvain, B1.30.24, Clos Chapelle-aux-Champs, 1200 Brussels, Belgium. ${ }^{3}$ Department of Epidemiology and Hygiene, Military Medical Academy of Sofia, Georgi Sofijsky blvd. 3, 1606 Sofia, Bulgaria. ${ }^{4}$ Bucharest Clinical Emergency Hospital, Calea Floreasca 8 Sector 1, Bucharest, Romania. ${ }^{5}$ Bukavu General Hospital, Université Catholique de Bukavu, P.O. Box 285, Bukavu, Democratic Republic of Congo.

\section{Acknowledgements}

This project was funded by the Department Management of Scientific and Technological Research of Defence (IRSD-RSTD; Royal High Institute for Defence) supporting research and development (Grants MED-20 and HFM14/8). We thank Elodie Carlier (IRSD-RSTD) for her contribution for this work.

\section{Compliance with ethical guidelines}

\section{Competing interests}

The authors declare that they have no competing interests.

\section{Ethic statements}

Ethical approval for the study was granted by the Institutional Review Board (IRB) of the Universite catholique Louvain. The study was carried out without an informed consent but yet complied with the World Health Organization and international guidelines on antibiotic surveillance for which no recommendation for an informed consent has been issued. In order to ensure confidentiality, samples were analyzed anonymously.

Received: 22 June 2015 Accepted: 23 July 2015

Published online: 12 August 2015

\section{References}

Al-Agamy MH, Shibl AM, Hafez MM, Al-Ahdal MN, Memish ZA, Khubnani H (2014) Molecular characteristics of extended-spectrum $\beta$-lactamaseproducing Escherichia coli in Riyadh: emergence of CTX-M-15-producing E. coli ST131. Ann Clin Microbiol Antimicrob 13:1-7

Ambroise J, Piette A, Delcorps C, Rigouts L, de Jong B, Irenge L (2013) AdvISERPYRO: amplicon identification using SparsE representation of PYROsequencing signal. Bioinformatics 29:1963-1969

Ambroise J, Deccache Y, Irenge L, Savov E, Robert A, Gala JL (2014) Amplicon identification using SparsE representation of multiplex PYROsequencing signal (AdvISER-M-PYRO): application to bacterial resistance genotyping. Bioinformatics 30:3590-3597 
Ambroise J, Butoescu V, Robert A, Tombal B, Gala JL (2015) Multiplex pyrosequencing assay using adviser-mh-pyro algorithm: a case for rapid and cost-effective genotyping analysis of prostate cancer risk-associated SNPs. BMC Med Genet 16:42

Bosshard PP, Zbinden R, Abels S, Böddinghaus B, Altwegg M, Böttger EC (2006) $16 \mathrm{~S}$ rRNA gene sequencing versus the API 20 NE system and the VITEK 2 ID-GNB card for identification of nonfermenting Gram-negative bacteria in the clinical laboratory. J Clin Microbiol 44:1359-1366

Bradford PA (2001) Extended-spectrum $\beta$-lactamases in the 21 st century: characterization, epidemiology, and detection of this important resistance threat. Clin Microbiol Rev 14:933-951

Bush K (2010) Alarming $\beta$-lactamase-mediated resistance in multidrug-resistant Enterobacteriaceae. Curr Opin Microbiol 13:558-564

Bush K (2013) The ABCD's of $\beta$-lactamase nomenclature. J Infect Chemother 19:549-559

Carter MW, Oakton KJ, Warner M, Livermore DM (2000) Detection of extendedspectrum $\beta$-lactamases in klebsiellae with the Oxoid combination disk method. J Clin Microbiol 38(11):4228-4232

Cohen Stuart J, Dierikx C, Al Naiemi N, Karczmarek A, Van Hoek AH, Vos P et al (2010) Rapid detection of TEM, SHV and CTX-M extended-spectrum betalactamases in Enterobacteriaceae using ligation-mediated amplification with microarray analysis. J Antimicrob Chemother 65:1377-1381

Cormican MG, Marshall SA, Jones RN (1996) Detection of extended-spectrum beta-lactamase (ESBL) producing strains by the Etest ESBL screen. J Clin Microbiol 34(8):1880-1884

D'Andrea MM, Arena F, Pallecchi L, Rossolini GM (2013) CTX-M-type $\beta$-lactamases: a successful story of antibiotic resistance. Int J Med Microbiol 303:305-317

Deccache Y, Irenge LM, Savov E, Ariciuc M, Trifonova A, Gergova I et al (2011) Development of a pyrosequencing assay for rapid assessment of quinolone resistance in Acinetobacter baumannii isolates. J Microbiol Methods 86:115-118

Drawz SM, Bonomo RA (2010) Three decades of beta-lactamase inhibitors. Clin Microbiol Rev 23:160-201

Drieux L, Brossier F, Sougakoff W, Jarlier V (2008) Phenotypic detection of extended-spectrum beta-lactamase production in Enterobacteriaceae: review and bench guide. Clin Microbiol Infect 14:90-103

Giske CG, Martinez-Martinez L, Cantón R, Stefani S, Skov R, Glupczynski Y et al (2013) EUCAST guidelines for detection of resistance mechanisms and specific resistances of clinical and/or epidemiological importance, EUCAST, 2012 [December]. Available from: https://www.eucast.org

Garrec H, Drieux-Rouzet L, Glomard JL, Jarlier V, Robert J (2011) Comparison of nine phenotypic methods for detection of extended-spectrum beta-lactamase production by Enterobacteriaceae. J Clin Microbiol 49:1048-1057

Gazin M, Paasch F, Goosens H, Malhotra-Kumar S (2012) Current trends in culture-based and molecular detection of ESBL- harboring and carbapenem-resistant Enterobacteriaceae. J Clin Microbiol 50:1140-1146

Giamarellou H (2005) Multidrug resistance in Gram-negative bacteria that produce extended-spectrum $\beta$-lactamases (ESBLs). Clin Microbiol Infect $11: 1-16$

Gibold L, Robin F, Tan RN, Delmas J, Bonnet R (2014) Four-year epidemiological study of extended-spectrum $\beta$-lactamase-producing Enterobacteriaceae in a French teaching hospital. Clin Microbiol Infect 20:O20-O26

Haanperä M, Huovinen P, Jalava J (2005) Detection and quantification of macrolide resistance mutations at positions 2058 and 2059 of the 23 S rRNA gene by pyrosequencing. Antimicrob Agents Chemother 49:457-460

Haanperä M, Forssten SD, Huovinen P, Jalava J (2008) Typing of SHV extendedspectrum beta-lactamases by pyrosequencing in Klebsiella pneumoniae strains with chromosomal SHV beta-lactamase. Antimicrob Agents Chemother 52:2632-2635

Ihaka R, Gentleman R (1996) R: a language for data analysis and graphics. J Comput Graph Stat 5(3):299-314

Jacoby GA (2009) AmpC beta-lactamases. Clin Microbiol Rev 22:161-182

Jacoby GA, Munoz-Price LS (2005) The new $\beta$-lactamases. N Engl J Med 352:380-391

Jarlier V, Nicolas MH, Fournier G, Philippon A (1988) Extended broad-spectrum beta-lactamases conferring transferable resistance to newer beta-lactam agents in Enterobacteriaceae: hospital prevalence and susceptibility patterns. Rev Infect Dis 10:867-878
Jones CH, Ruzin A, Tuckman M, Visalli MA, Petersen PJ, Bradford PA (2009) Pyrosequencing using the single-nucleotide polymorphism protocol for rapid determination of TEM- and SHV-type extended-spectrum betalactamases in clinical isolates and identification of the novel beta-lactamase genes blaSHV-48, blaSHV-105, and blaTEM-155. Antimicrob Agents Chemother 53:977-986

Kong KF, Schneper L, Mathee K (2009) Beta-lactam antibiotics: from antibiosis to resistance and bacteriology. APMIS 118:1-36

Leinberger DM, Grimm V, Rubtsova M, Weile J, Schröppel K, Wichelhaus TA et al (2010) Integrated detection of extended-spectrum-beta-lactam resistance by DNA microarray-based genotyping of TEM, SHV, and CTX-M genes. J Clin Microbiol 48:460-471

Leverstein-van Hall MA, Fluit AC, Paauw A, Box AT, Brisse S, Verhoef J (2002) Evaluation of the Etest ESBL and the BD Phoenix, VITEK 1, and VITEK 2 automated instruments for detection of extended-spectrum beta-lactamases in multiresistant Escherichia coli and Klebsiella spp. J Clin Microbiol 40:3703-3711

Livermore DM, Canton R, Gniadkowski M, Nordmann P, Rossolini GM, Arlet G et al (2007) CTX-M: changing the face of ESBLs in Europe. J Antimicrob Chemother 59:165-174

Longo MC, Berninger MS, Hartley JL (1990) Use of uracil DNA glycosylase to control carry-over contamination in polymerase chain reactions. Gene 93(1):125-128

March A, Aschbacher R, Dhanji H, Livermore DM, Böttcher A, Sleghel F et al (2010) Colonization of residents and staff of a long-term-care facility and adjacent acute-care hospital geriatric unit by multiresistant bacteria. Clin Microbiol Infect 16:934-944

Munier GK, Johnson CL, Snyder JW, Moland ES, Hanson ND, Thomson KS (2010) Positive extended-spectrum-beta-lactamase (ESBL) screening results may be due to AmpC beta-lactamases more often than to ESBLs. J Clin Microbiol 48:673-674

Naas T, Oxacelay C, Nordmann P (2007) Identification of CTX-M-type extended-spectrum-beta-lactamase genes using real-time PCR and pyrosequencing. Antimicrob Agents Chemother 51:223-230

Nijhuis R, van Zwet A, Cohen Stuart J, Weijers T, Savelkoul P (2012) Rapid molecular detection of extended-spectrum $\beta$-lactamase gene variants with a novel ligation-mediated real-time PCR. J Med Microbiol 61:1563-1567

Owens RC Jr, Lautenbach E (2008) Antimicrobial resistance: problem pathogens and clinical countermeasures. Informa Healthcare, New York

Oxacelay C, Ergani A, Naas T, Nordmann P (2009) Rapid detection of CTX-Mproducing Enterobacteriaceae in urine samples. J Antimicrob Chemother 64:986-989

Paterson DL (2006) Resistance in gram-negative bacteria: Enterobacteriaceae. Am J Med 34:S20-S28

Paterson DL, Bonomo RA (2005) Extended-spectrum beta-lactamases: a clinical update. Clin Microbiol Rev 18:657-686

Perez F, Endimiani A, Hujer KM, Bonomo RA (2007) The continuing challenge of ESBLs. Curr Opin Pharmacol 7:459-469

Pfaller MA, Segreti J (2006) Overview of the epidemiological profile and laboratory detection of extended-spectrum beta-lactamases. Clin Infect Dis 42:153-163

Pitout JD, Laupland KB (2008) Extended-spectrum beta-lactamase-producing Enterobacteriaceae: an emerging public-health concern. Lancet Infect Dis 8:159-166

Pitout JD, Sanders CC, Sanders E (1997) Antimicrobial resistance with focus on beta-lactam resistance in Gram-negative bacilli. Am J Med 103:51-59

Polsfuss S, Bloemberg GV, Giger J, Meyer V, Böttger EC, Hombach M (2011) Practical approach for reliable detection of AmpC beta-lactamase-producing Enterobacteriaceae. J Clin Microbiol 49:2798-2803

Poole K (2004) Resistance to $\beta$-lactam antibiotics. Cell Mol Life Sci 61:2200-2223

Robin F, Delmas J, Schweitzer C, Bonnet R (2008) Evaluation of the Vitek-2 extended-spectrum beta-lactamase test against non-duplicate strains of Enterobacteriaceae producing a broad diversity of well-characterised beta-lactamases. Clin Microbiol Infect 14:148-154

Rossolini GM, D'Andrea MM, Mugnaioli C (2008) The spread of CTX-M-type extended-spectrum beta-lactamases. Clin Microbiol Infect 14:33-41

Rupp ME, Fey PD (2003) Extended spectrum beta-lactamase (ESBL)-producing Enterobacteriaceae: considerations for diagnosis, prevention and drug treatment. Drugs 63:353-365 
Stürenburg E, Mack D (2003) Extended-spectrum beta-lactamases: implications for the clinical microbiology laboratory, therapy, and infection control. J Infect 47:273-295

Tsakris A, Poulou A, Themeli-Digalaki K, Voulgari E, Pittaras T, Sofianou D et al (2009) Use of boronic acid disk tests to detect extended- spectrum beta-lactamases in clinical isolates of KPC carbapenemase-possessing Enterobacteriaceae. J Clin Microbiol 47:3420-3426
Vandercam B, Jeumont S, Cornu O, Yombi J, Lecouvet F, Lefèvre P et al (2008) Amplification-based DNA analysis in the diagnosis of prosthetic joint infection. J Mol Diagn 10:537-543

Wintermans BB, Reuland EA, Wintermans RG, Bergmans AM, Kluytmans JA (2013) The cost-effectiveness of ESBL detection: towards molecular detection methods? Clin Microbiol Infect 19:662-665

\section{Submit your manuscript to a SpringerOpen ${ }^{\circ}$ journal and benefit from:}

- Convenient online submission

- Rigorous peer review

- Immediate publication on acceptance

- Open access: articles freely available online

- High visibility within the field

- Retaining the copyright to your article

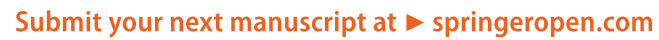

